\title{
Correction: Dynamics of Actin Waves on Patterned Substrates: A Quantitative Analysis of Circular Dorsal Ruffles
}

\section{The PLOS ONE Staff}

Due to a typesetting error, Fig. 2 is missing an essential variable (the Greek $\tau$ "tau") in three places. The publisher apologizes for the error. Please view the complete, correct Fig. 2 here.

\section{G openaccess}

Citation: The PLOS ONE Staff (2015) Correction: Dynamics of Actin Waves on Patterned Substrates: A Quantitative Analysis of Circular Dorsal Ruffles. PLoS ONE 10(3): e0119746. doi:10.1371/journal. pone. 0119746

Published: March 25, 2015

Copyright: $\odot 2015$ The PLOS ONE Staff. This is an open access article distributed under the terms of the Creative Commons Attribution License, which permits unrestricted use, distribution, and reproduction in any medium, provided the original author and source are credited. 
A

B
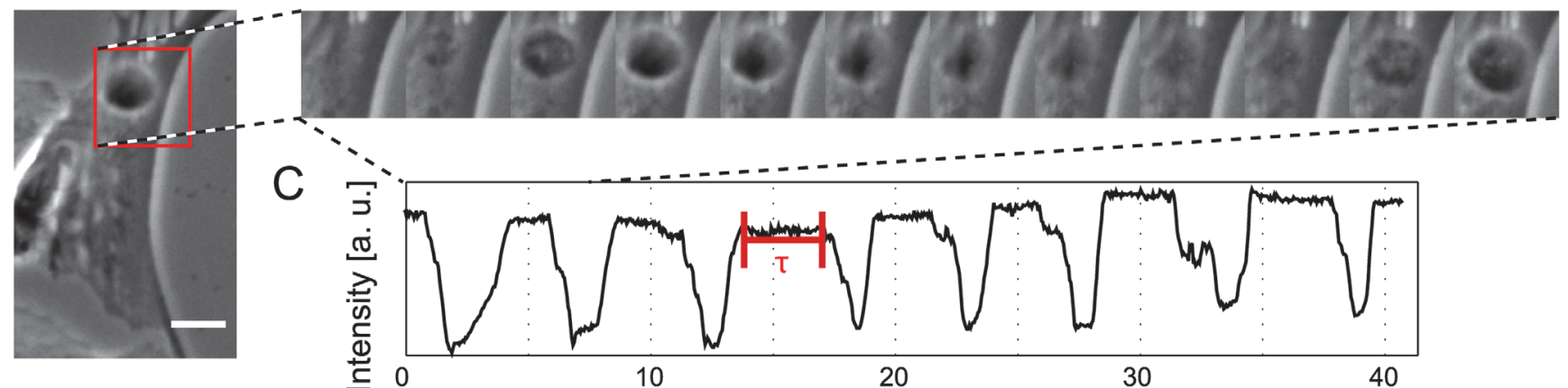

尚
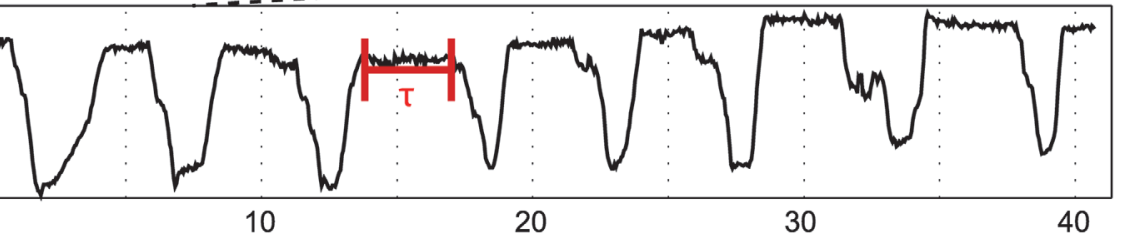

$t[\mathrm{~min}]$

D

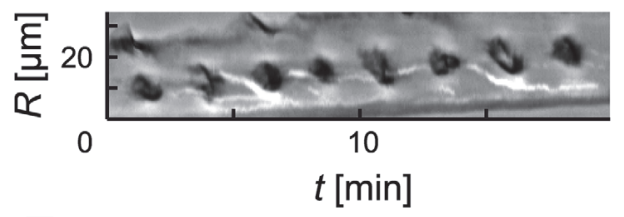

$E$

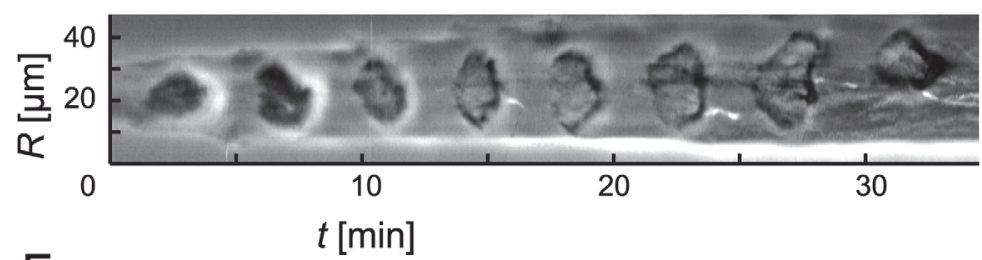

G

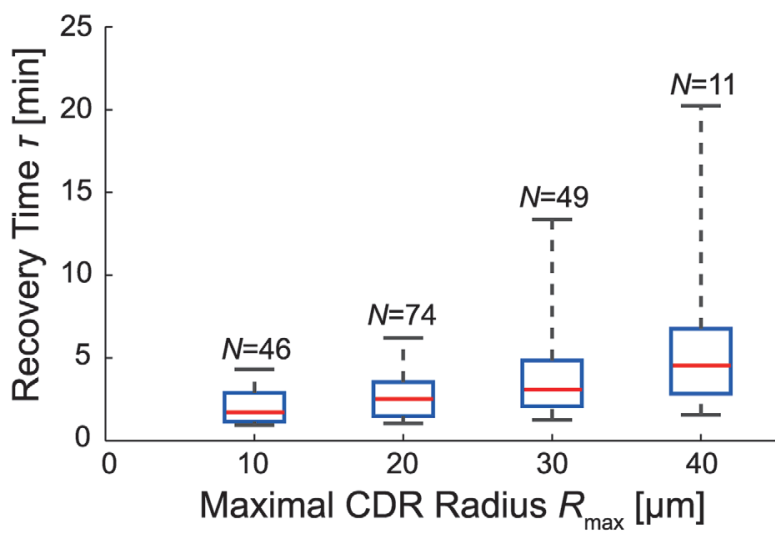

F

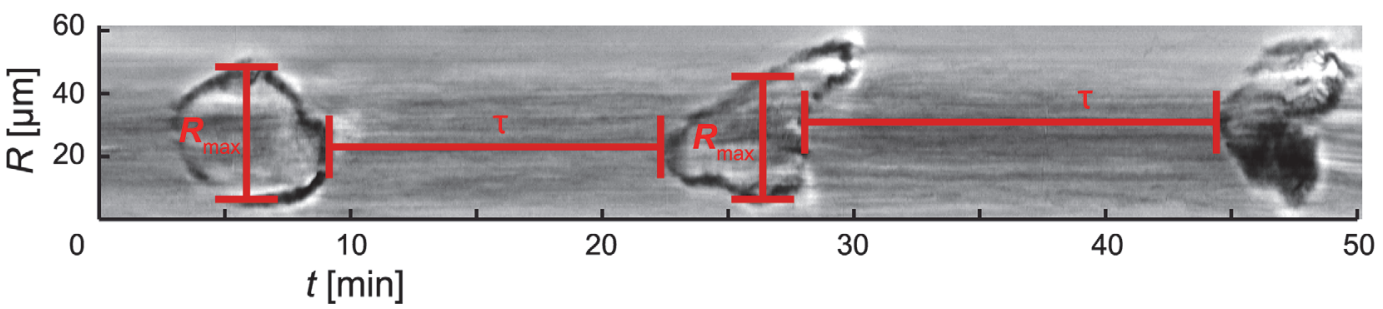

Fig 2. Oscillatory reappearing CDRs. $(A)$ CDRs under spatial confinement exhibit oscillatory patterns of pulsating re-appearance (scale bar: $25 \mu \mathrm{m}$, full sequence: S5 Movie). (B) Stills from the region of interest highlighted red in the time-lapse sequence $A(\Delta t=36 \mathrm{~s})$. (C) A plot of the minimal intensity value of the ROI in $A$ as a function of time shows CDR events as negative peaks and CDR-free periods, corresponding to the recovery time T, as plateaus of high intensity. The ROI was smoothed with a Gaussian with $\sigma=2 \mu \mathrm{m}$ prior to intensity sampling. $(D-F)$ Kymographs of CDRs taken along lines crossing CDR origins (see Fig. 4 Afor illustration) show both the recovery time t between successive events and their radial extension $R_{\max }$ (cells not shown). (G) The recovery times increase with CDR size. The data was binned in $R_{\max }$-direction (box width: $10 \mu \mathrm{m}$ ) and plotted as boxes with whiskers (red lines: median, upper box edge: 75 th percentile, lower box edge: 25 th percentile). $N$ values denote the number of observations. Note that oscillatory behavior was rare for large CDRs.

doi:10.1371/journal.pone.0119746.g001

\section{Reference}

1. Bernitt E, Koh CG, Gov N, Döbereiner H-G (2015) Dynamics of Actin Waves on Patterned Substrates: A Quantitative Analysis of Circular Dorsal Ruffles. PLoS ONE 10(1): e0115857. doi:10.1371/journal. pone.0115857 PMID: 25574668 